\title{
Suicide, Mental Illness and Maori People
}

\author{
Said Shahtahmasebi ${ }^{1,2,3 *}$, Bernadette Cassidy ${ }^{2,4}$ \\ ${ }^{1}$ Centre for Health and Social Practice, Wintec, Hamilton, New Zealand \\ ${ }^{2}$ The Good Life Research Centre Trust, Christchurch, New Zealand \\ ${ }^{3}$ Voluntary Faculty, University of Kentucky, Lexington, KY, USA \\ ${ }^{4}$ NZ Spinal Trust, Allan Bean Centre, Burwood Hospital, Christchurch, New Zealand \\ Email: ${ }^{*}$ radisolevoo@gmail.com
}

Received 11 June 2014; revised 27 July 2014; accepted 12 August 2014

Copyright (C) 2014 by authors and Scientific Research Publishing Inc.

This work is licensed under the Creative Commons Attribution International License (CC BY). http://creativecommons.org/licenses/by/4.0/

(c) (i) Open Access

\begin{abstract}
Globally, authorities and "experts" continually assert that suicide is a major public health concern and it is preventable. However, decades of suicide prevention strategies have seen "more of the same" action plans but no change in the upward suicide trend. Therefore, the current suicide prevention model is less relevant to indigenous and minority populations with a high suicide rate. Current suicide statistics for Maori, New Zealand's indigenous population are unacceptably high. The Maori suicide rate is about 19 per 100,000 roughly averaging about 104 deaths per year over the last six years. Maori claim that before colonisation suicide was non-existent. There is certainly evidence to support such a claim. e.g., historical suicide data suggested that the number of Maori youth suicide deaths was less than five until the 1970s and 1980s. Maori now have the dubious honour of having the highest rates of mortality and morbidity outcomes, including higher rates of suicide. Neither Maori nor the authorities responded with an action plan when suicide numbers spiked in 1960 and 1967. Subsequently, the number of suicides rose sharply to over one hundred where they stayed. It is plausible that exposure to Western ideals as well as social insensitivity to Maori beliefs and needs may have led to a cultural dealignment during the1960s and 1970s. This cultural shift also may be due to the application of a Western model of suicide prevention based on mental illness. The Western model does not work in preventing suicide and conflicts with indigenous cultures.
\end{abstract}

\section{Keywords}

Maori Suicide, Mental Health, Public Health, Cultural Insensitivity

\section{Introduction}

The eleventh world suicide prevention day was observed in September 2013. A quick search of the internet re-

Corresponding author. 
vealed that the world suicide prevention day, September 10, was acknowledged with a number of conferences and meetings. The question arises—-what is meant by world suicide prevention day? What does the world do on this day that it does not do the rest of the year? What have we gained from marking a day for suicide prevention? Suicide trends follow a cyclic pattern: maybe on this day suicide will be prevented or at least the cycle will be broken. But our suicide prevention strategies along with health, social and economic policies ensure that suicide does not rest nor does it sleep [1]. Suicide rates show an increasing trend [1], more so for indigenous populations and minority groups over recent years [2].

We are faced with a number of problems. First, symbolism (conferences to mark a suicide prevention day) does not prevent suicide but lead to suicide prevention action plans that provide "more of the same" [2] year after year and cycle after cycle. For example, suicide data along with mortality, morbidity and prescription data suggest that decades of suicide prevention and intervention based on mental illness does not work [2]. However, in New Zealand the authorities claim success when facing a cycle down-turn and demand more funding to provide more mental health services. Conversely, when facing a cycle up-turn, they explain that suicide is a complex public health issue with lots of complex socio-economic risk factors and demand more funding in order to provide outreach mental illness health services to all sectors of society.

Second, the current mental illness model of suicide prevention recommends that public and frontline care workers look for signs (e.g. depression) and refer to mental health services. Several issues arise here : 1) There is no statistical evidence to support a causal link between mental illness and suicide; 2) mental health services treat the "patient" for depression or the symptoms that are presented but not for suicide; 3) this approach is not prevention because an event has occurred. Prevention is avoiding an individual getting to the stage where they may feel suicidal; 4) this approach misses out the majority of people who do not exhibit symptoms or are good at hiding them; 5) decades of uncritical acceptance that mental illness causes suicide has made it difficult for the "experts" to view suicide outside of a mental illness framework. For example, Mulder, a staunch supporter of the medical model has recently come to the conclusion that the medical model does not work [3]. However, his alternative of risk management instead of risk assessment [3] is no different to the medical model that he now believes does not work! The reason the medical model does not work is because there is no significant causal link between mental illness and suicide. Therefore, whether assessing or managing symptoms of mental illness as suicide risk will only affect those symptoms but may not prevent suicide. This is why suicidal patients complete suicide while receiving psychiatric care or following discharge after completing psychiatric treatment.

Third, quite apart from the fact that the medical model does not prevent and stop suicide, the approach is often in conflict with cultural traditions and systems of belief. A historical discussion of the development of modern psychiatry and its relation to suicide is beyond the scope of this paper. Suffice it to say it is possible that a system that seeks to explain human behaviour purely based on chemical and electrical activity of the brain will clash with cultural beliefs and traditions which have allowed people with mental illness to be fully integrated members of their community without labelling them as mentally ill and incarcerate them.

Fourth, the domination of cultural traditions by a dominant culture attenuates any influence and impact from those traditions in dealing with mental health issues. Thus, neither approach will prevent or stop suicide.

The Western approach to suicide prevention is narrow, exclusive, one-dimensional and one-way (i.e. topdown) which makes it insensitive not only to indigenous people but other cultures. In this paper, we raise some important issues relevant to suicide prevention for Maori people in New Zealand, in order to stimulate debate and provide appropriate suicide prevention action plans.

\section{Background}

Suicide is an act of violence against oneself and the person committing suicide dies alone. Suicide has a huge impact on family and friends which lasts for decades. The decision to end one's own life not only has a profound impact on one's community but it is also making a harsh statement about that community/society. Suicide occurs at a young age or during adolescence, which suggests that the communities concerned have lost their way not the suicide victim. In other words, communities are too pre-occupied with the politics of daily living and survival to such an extent that they do not hear cries for help. As a result society has found it easier or convenient to treat calls for help as though they were cracks in a wall which can then be filled with mental illness remedies.

Mental illness is far too simplistic a term to explain suicide let alone prevent it.

This should be a wake up call for all of us. The current simplistic approach of using mental illness as the 
cause, in particular, for the purpose of preventing suicide, has not explained nor has it prevented suicide especially in those communities that traditionally have had a very low suicide rate.

Most suicide prevention strategies and initiatives are based on the assumption that the root cause of suicide is depression or mental illness. Attempts to prevent suicide based on this assumption are only interventions, i.e. looking for warning signs (an event has occurred) followed by referral to mental health services (medical intervention: treating the symptoms). The warning signs include depression, previous attempts, mental illness, and so on. In other words, depression and mental illness are addressed by mental health services but not "suicide" per se.

Perhaps, the fact that symptoms of mental illness, which may or may not exist, are treated in order to intervene and stop suicide makes this approach insensitive to indigenous and other cultures. This insensitivity makes the service inappropriate and is responsible, at least in part, for the stigma attached to suicide and mental illness thus steering people away from mental health services. For example, between two-thirds and three-quarters of all suicide cases do not come into contact with mental health services, the remainder who received psychiatric care then go on to complete suicide [4]-[6].

The problem with suicide research is that the main provider of information is no longer alive and most of the "knowledge" that is available is subjective and based on hearsay after suicide has occurred. Despite a lack of the evidence, the public mind set is well conditioned towards mental illness (depression) being the cause of suicide.

At the centre of each suicide is a human being, and suicide occurs regardless of culture, sex and age. Therefore, suicide is the outcome of a decision making process. Processes are dynamic by nature. Temporal dependencies may affect the process of decision making, for example, in youth and adolescents, $30 \%$ of suicides are impulsive same day incidents [7], while on the other hand adults take time to plan suicide over a longer period.

Suicide prevention makes more sense when suicide is viewed as a decision outcome which then forces the consideration of everything that influences the process of decision making. Experience suggests that people with suicidal tendencies are treated the same as those with signs of a mental illness, i.e., a diagnosis is made which involves prescribing medication, such as antidepressants. Therefore the health care system fails to address suicide; the decision process, and as a result the person.

Suicide prevention must be based on understanding the person first in order to inform their process of decision making [8]. In other words, a "person centred" approach is necessary. The following excerpt from a physician's diary [9] retold here, demonstrates the application of the concept of treating the "whole" person:

A patient had exhibited psychological symptoms and had refused to eat believing that he possessed mythical and supernatural powers. He insisted that he wanted to shift the Rocky Mountains on the outskirts of the city with his bare hands. His family had consulted well-known consultants and specialists; and all manner of tests had been conducted and treatments prescribed. By the time I was contacted, the patient was in bad shape; he was emaciated and skeletal, and was tied down to the bed for his own safety. The family explained that they had tried everything and that I was their last hope. After checking the patient's history, I asked for the patient to be untied and told the patient that I had come for his help to shift the Rocky Mountains and had heard that he (the patient) was the only one that could help. To the horror of the family I helped the patient to get organized and we walked towards the front door. I reached for the door handle, but before opening it, I turned to the patient and said "let's shake on our new partnership!” I squeezed the patient's hand so hard that the patient winced in pain. I persuaded the patient that since his hands are important for this mission, he should have something to eat and then try again the next day. The patient voluntarily went back to his room and waited to be fed. In the meantime, I wrote up a comprehensive prescription focused on the patient's dietary requirements and gave it to the family. This same pattern of events continued the next day and the next and the next and so on. On what became my final visit, after examining the patient I said that he was now strong enough to go and help shift the Rocky mountains to which the patient responded "are you crazy doctor! Why would I want to do that?"

A western style medical model is in direct conflict with the philosophy of a person-centred approach of some indigenous groups. e.g. see [10]. And it is this conflict that makes health services insensitive to the needs of indigenous and minority groups as well as the general population, and it is also the cause of social disharmony and dealignment, e.g. low service uptake and inequalities in health and social outcomes [11]-[13].

\section{Methodology}

Maori history is encapsulated in stories that have been passed down the generations, and in recent years attempts 
have been made to collect and archive historical evidence. Maori, the indigenous population of New Zealand claim that suicide and western diseases were unheard of before colonisation. Certainly, Maori stories provide some support for such a claim, but the stories do not shed any light on whether zero suicide was due to Maori belief systems, culture and practices, or, tribal wars. However, there are at least two major issues that have arisen. First, the introduction of suicide into Maori culture by the white man, and second, the inappropriateness and insensitivity of solutions offered by the white man.

A secondary data source, namely, mortality records were used to explore Maori suicide. Mortality records by ethnicity go back only as far as 1948 but do provide some clues about the dynamics of Maori suicide post colonisation.

Excerpts from case studies of suicide survivors, i.e. family and friends who have lost a loved one to suicide [2] [14]-[16] are also used to explore the Maori view of cultural sensitivity and the appropriateness of mental health services.

\section{Suicide}

In New Zealand, Maori have the worst health outcomes, both in terms of morbidity and mortality and service uptake [17]. For example, the incidence and prevalence of obesity, diabetes, heart disease, youth suicide, smoking and drinking are higher in Maori than the general population. The causes for these undesired outcomes is lumped into one category and labelled as being Maori, e.g. lack of education about services, lack of access to services, overcrowding, customs, traditions and culture. For example a recent report claims that housing overcrowding in Maori and Pacific populations is responsible for a number of infectious diseases (http://www.stuff.co.nz/national/health/8768249/House-overcrowding-disease-fears). This study is based on an international systematic review incorporating New Zealand census data and wrongly ignores temporal dependencies and the tradition of extended family which is integral to this group's way of life, and unwisely concludes overcrowding as the main causal factor. Clearly, in small households it is likely that fewer people become "infected" than in larger households but this does not mean that overcrowding is the cause of infectious diseases.

Poor health outcomes and poor service uptake by Indigenous groups is interpreted as "unmet needs" as opposed to the insensitivity of the services in question. As a result, the question of "why needs go unmet" is presumed to be the result of socio-economic and environmental conditions making access to services problematic for these groups (e.g. see [18]).

Maori oral history and stories claim that suicide in Maori was unheard of in traditional times before colonisation by the Europeans (TeKaniKingi 2005, http://www.massey.ac.nz/massey/fms/Te\%20Mata\%200\%20Te\%20Tau/Publications\%20-\%20Te\%20Kani/T\% 20Kingi\%20Indigenety\%20and\%20Maori\%20mental\%20health.pdf). Certainly, as is shown in Figure 1, the number of deaths by suicide in young people was well under 5 per year since records began until 1974. The number of youth suicide reached 5 in 1974, 75, 77, 80 and 81, but in 1984 the numbers climbed to 6 and continued to increase. The total number of deaths by suicide, however, (Figure 1) went up to 11 as early as 1961. There were two more warnings in 1967 and 1970 when the total number of suicides reached double digits. The increases in suicide should have been heeded as a warning and contingency plans put in place. Clearly, no prevention plans had been adopted since the first warning, and/or, if there were any suicide prevention plans none had worked as from 1973 suicide numbers began to climb reaching 43 deaths in 1993. In 1995 a new definition for ethnic origin(s) was adopted, as a result suicide numbers and rates post 1995 are not comparable with pre 1995 data. However the impact of this change on suicide numbers is quite dramatic, see Figure 2.

\section{Discussion and Conclusions}

The insensitivity of the current "mental illness based suicide prevention" can be explored through the following case. For full details see [15]. This is the story of an eighteen year old, a New Zealander of European ancestry, who completed suicide after a number of attempts, away from home, family and friends in another country. The case was described by his mother as a high achiever and was involved in many activities, and played violin in the youth orchestra. The case's first attempt was at sixteen and as a result had about six to eight sessions with mental health services. Their diagnosis was that the suicide attempt was carried out on impulse and that the case wouldn't do it again. The case's reason for the attempt was that he/she had been worrying about failing an English test taken the day before: 


\section{Number of Maori Suicides Pre 1995}

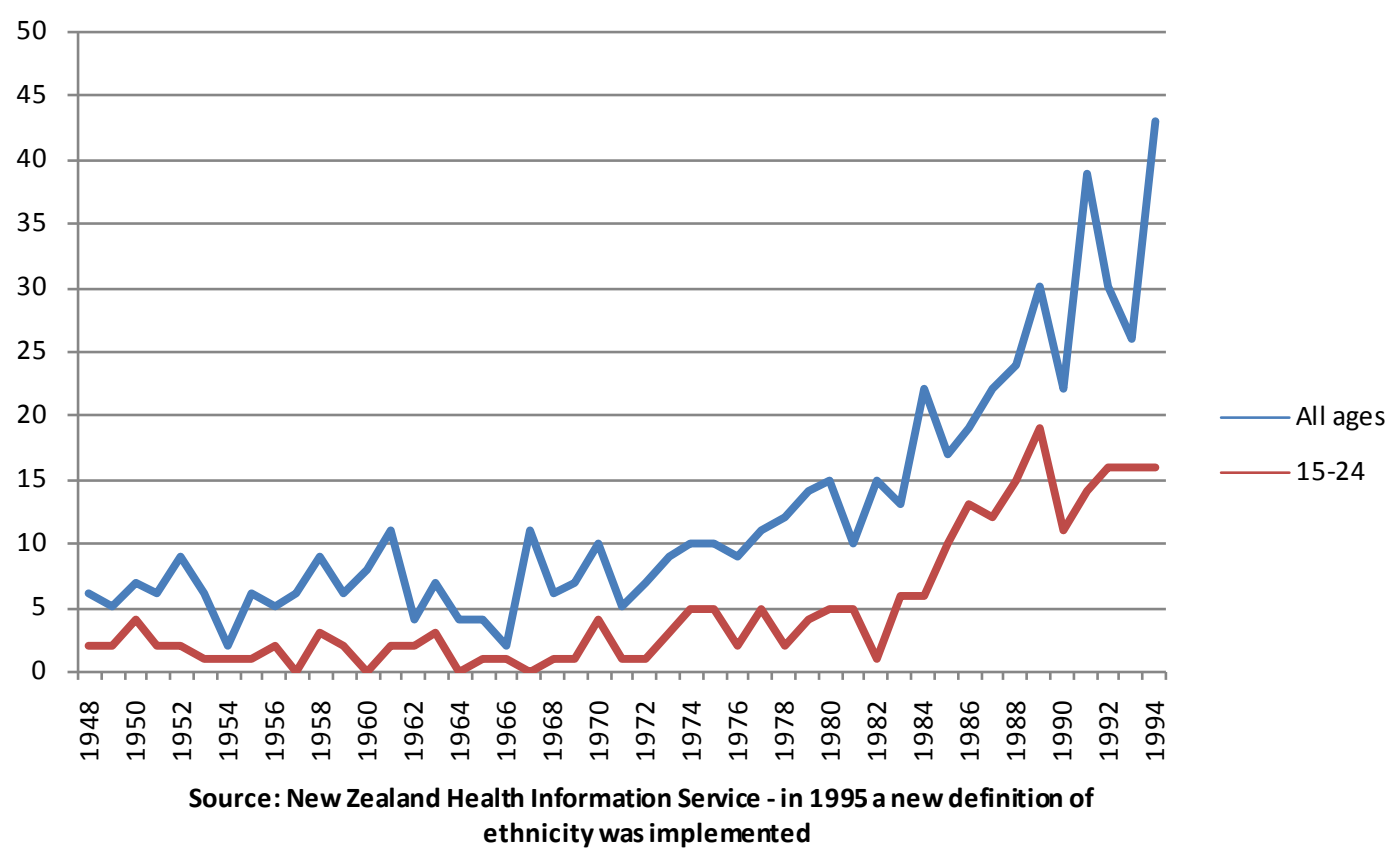

Figure 1. Number of Maori suicides pre 1995.

Number of Maori Suicides Pre and Post 1995

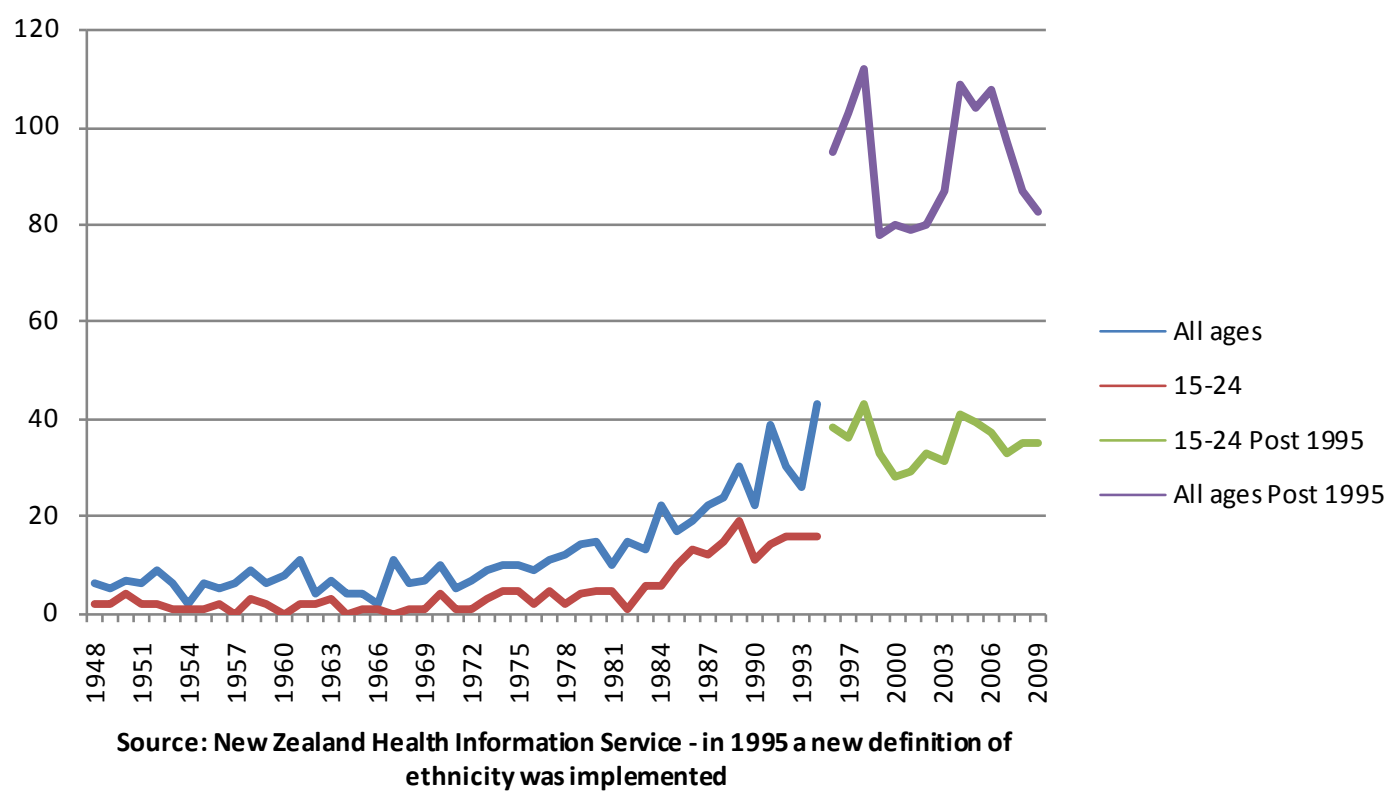

Figure 2. Number of Maori suicides pre and post 1995.

"With a bit of hindsight, that to me should have raised some alarm bells in the psychiatrist's mind, because it is a very unrealistic reason for committing suicide. It really isn't a good reason to commit suicide, but anyhow. So they said well it's an impulse control problem, and X now understood why X acted the way s/he did, and X knew what to do next time if s/he ever felt like that, and X was good-X wouldn't do it again. $\mathrm{X}$ was good for about six months, no medications, no nothing like that, and then $\mathrm{X}$ tried again, same method, all of X's methods except for the last two involved pills.” 
This time the case was taken to psychiatric services in the main city but was seen by a different person, and had two to three sessions with the same outcome: that it was an impulse and wouldn't happen again.

So many trivial reasons for wanting to end one's life should have raised concerns with the psychiatrists. The case's mother asserts that on each occasion the case was seen by a different psychiatrist who appeared not to have read the case's file. The mother argues that each attempt was treated as a new case by psychiatric services as though there were no previous history, while at the same time they did not appear to take on-board the verbal history of the case. This explains the reason for mental health services delivering the same medical outcome: "suicide attempt on impulse" and "it wouldn’t happen again”, but it did happen again:

“And every single time they were saying $\mathrm{X}$ was fixed, you won't do it again, and every single time $\mathrm{X}$ did it again the message $X$ was getting was well you're wrong, you're lying to me and you don't know what you're talking about because I did do it again, I did feel like that again. They were saying to X you won't feel like this again, you won't, next time you'll be able to cope, but X wasn't able to cope. As far as I'm concerned the only message mental health services were giving $X$ was that we really don't know how to help you."

Spiritualism is important in Maori in dealing with life matters and events. Exposure to the dominant mainstream culture has attenuated Maori mainstream practices. The following excerpt from a suicide survivor's story of how she lost her eldest child to suicide can provide some insight into Maori spiritual beliefs:

"Well now, in our Whānau we're quite spiritual people and one of my cousins used to say to me her boy, when $\mathrm{X}$ used to come back, $\mathrm{X}$ used to tell this cousin that $\mathrm{X}$ used to hear things. $\mathrm{X}$ was hearing voices. And his cousin would say to $X$ it's all right. Because for us that's like hearing our tepuna.

Yeah. And actually, oh I forgot this part too-when $\mathrm{X}$ was young, when $\mathrm{X}$ was about eight, $\mathrm{X}$ would see things at home. $\mathrm{X}$ and my second oldest used to see things at home. I used to tell $\mathrm{X}$ stop being silly, go to sleep, but my second oldest still remembers what it looked like and where it was now. It's because they were, I don't know, seers?

Yeah, $\mathrm{X}$ and my oldest one. They used to say mummy, mummy it's in the wardrobe. I used to tell them go to sleep, coz they were both in the same room. And I never believed them. I never believed them. I just thought oh they're just being naughty. Well when my mother in law passed away, we have a tohunga that we go to. Well my husband wasn't settling very well after his mum died so we went to see this tohunga and we took our Whānau with us, and tohunga said to us oh there's something in your house, your kids have seen it when they were little. And they looked at me like told you so. Yeah, they gave me that look like we told you. Coz the tohunga picked it up.”

Traditional Maori consider it a gift to be able to hear voices or see things that ordinary humans cannot see or hear. Therefore, traditions in Maori were developed to control the gift and embrace the gifted through social positioning of tohunga (priest, medium, see

http://www.teara.govt.nz/en/traditional-maori-religion-nga-karakia-a-te-maori/page-2).

A system of beliefs that embraces and accepts mental diversity and considers it a gift will clash with a western system of beliefs that considers mental diversity as an illness and medicates and incarcerates people with these symptoms [19] [20]. Centuries of traditions have in place the social structure and social codes for the community to deal with mental diversity. Thus, the belief system is sustained in the community. The exposure of indigenous people to western ideals and medicine has had a major impact on their beliefs creating confusion for generations to come. Therefore, the Maori system of beliefs cannot function effectively in the absence of traditions with regard to child rearing-perhaps Maori should develop the art of dealing with the "gift" when the gifted person does not want it. Nowadays, it is likely that, for indigenous people, both belief systems are eroded sufficiently so that both are treated as an off-the-shelf solution, which can be observed from the following excerpt from the same suicide survivor:

"With X, we tried to embrace our spiritual, the holistic side, with the western Pakeha medicine. We tried to embrace both. But X, used to say it's not going to work. It's not working mum, $X$ used to tell me, my medicine's not working. $X$ even used to tell the doctor I have a gift. Coz the tohunga had told $X$ that $X$ had a gift, so in X's mind that was what was wrong with $X$, it was the gift. And then after a while, even with the 
doctors giving different medication X was just yeah it's working but it wasn't, X was still hearing things.”

The dilemma that bedevils suicide prevention for indigenous populations is that having brought suicide into New Zealand the Europeans must now provide the solution. But, a lack of understanding of suicide coupled with cultural differences, as described above, has meant that a solution is beyond reach. As discussed in the introduction and elsewhere [1] the western model of suicide prevention does not work for the general population and Maori belief systems are without the traditional protection needed for it to have any impact.

The authorities often interpret the problem as one of increasing service uptake by indigenous populations. For example, one of the ways of reaching out to communities isolated through cultural and/or geographical differences is to train indigenous people in suicide prevention [19] [20]. Such approaches, under current suicide philosophy create more problems. Training indigenous people and/or front line service providers with a Western approach, i.e. to look for signs and refer to mental health services, does not incorporate cultural sensitivity, nor is it a grassroots approach to suicide prevention. In other words, this policy is one of Maori providing the dominant culture's suicide prevention for Maori. Besides, the evidence suggests that this approach is very expensive in terms of lives lost and resources; ultimately it does not work [1]. It simply provides a different name for the same model that provides "more of the same" but at much higher social and economic costs each year.

At one of the conferences marking the global suicide prevention day, a Maori presenter, herself, a suicide survivor, provided the following explanation: for Māori Whānau, another very real reason why some do not address suicide is because of Whakamaa. Whakamaa is often interpreted as shame, shyness or embarrassment. But it has much a deeper meaning than this. For someone like my husband whose world was totally, totally Māori, Whakamaa meant that when he was assessed by two Pākehā (non-Maori) clinicians three weeks before he took his life, he told them everything he thought they needed to hear-as opposed to what he needed in order to be heard. He would never have spoken with them about his visits from his tipuna (female ancestor) and nor should he. But understanding these beliefs does not fit within Western psychiatry or psychology, and so becomes a natural barrier to mainstream support [21].

A much better approach to suicide prevention is to adopt a grassroots approach which is inclusive of all cultures and will compliment Maori culture perfectly [1].

\section{References}

[1] Shahtahmasebi, S. (2013) De-Politicizing Youth Suicide Prevention. Frontiers in Pediatrics, 1, 8.

[2] Shahtahmasebi, S. (2013) Indigenous Populations and Suicide Prevention. International Public Health Journal, 6, Forthcoming.

[3] Mulder, R. (2013) Problems with Suicide Risk Assessment. Australian and New Zealand Journal of Psychiatry, 45, 605-607. http://dx.doi.org/10.3109/00048674.2011.594786

[4] Shahtahmasebi, S. (2003) Suicides by Mentally Ill People. Scientific World Journal, 3, 684-693. http://dx.doi.org/10.1100/tsw.2003.51

[5] Shahtahmasebi, S. (2005) Suicides in New Zealand. Scientific World Journal, 5, 527-534. http://dx.doi.org/10.1100/tsw.2005.74

[6] Hamdi, E., Price, S., Qassem, T., Amin, Y. and Jones, D. (2008) Suicides Not in Contact with Mental Health Services: Risk Indicators and Determinants of Referral. Journal of Mental Health, 17, 398-409. http://dx.doi.org/10.1080/09638230701506234

[7] Suicide Prevention Resource Center (2007) Youth Suicide: Findings from a Pilot for the National Violent Death Reporting System.

http://www.sprc.org/library resources/items/youth-suicide-findings-pilot-national-violent-death-reporting-system

[8] Ventegodt, S., Clausen, B. and Merrick, J. (2006) Clinical Holistic Medicine: The Case Story of Anna. III. Rehabilitation of Philosophy of Life during Holistic Existential Therapy for Childhood Sexual Abuse. The Scientific World Journal, 6, 2080-2091. http://dx.doi.org/10.1100/tsw.2006.338

[9] Shahtahmasebi, M. (1985) Treatment for a Diagnosis or Diagnosing a Treatment. Personal Communication.

[10] Sherwood, J. and Edwards, T. (2006) Decolonisation: A Critical Step for Improving Aboriginal Health. Contemporary Nurse, 22, 178-190. http://dx.doi.org/10.5172/conu.2006.22.2.178

[11] Bailey, J., Veitch, C., Crossland, L. and Preston, R. (2006) Developing Research Capacity Building for Aboriginal \& Torres Strait Islander Health Workers in Health Service Settings. Rural Remote Health, 6, 556.

[12] Incayawar, M. (2007) Indigenous Peoples of South America-Inequalities in Mental Health Care. In: Bhui, K. and Bhu- 
qra, D., Eds., Culture and Mental Health—A Comprehensive Textbook, Hodder Arnold, London, 185-190.

[13] McFarland E, Dalton M, Walsh D. (1989) Ethnic Minority Needs and Service Delivery: The Barriers to Access in a Glasgow Inner-City Area. Journal of Ethnic and Migration Studies, 15, 405-415. http://dx.doi.org/10.1080/1369183X.1989.9976128

[14] Shahtahmasebi, S. and Aupouri-Mclean, C. (2011) Bereaved by Suicide. Primary Health Care. http://omicsgroup.org/journals/bereaved-by-suicide-2167-1079.1000101.php?aid=2953

[15] Shahtahmasebi, S. and Merrick, J. (2013) Suicide from a Public Health Perspective. Nova, New York, in Press.

[16] Shahtahmasebi, S. and Smith, L. (2013) Has the Time Come for Mental Health Services to Give up Control? Journal of Alternative Medicine Research, 6, in Press.

[17] Ministry of Health (2013) The Health of Maori Adults and Children. http://www.health.govt.nz/publication/health-maori-adults-and-children

[18] Hunter, E. (2007) Disadvantage and Discontent: A Review of Issues Relevant to the Mental Health of Rural and Remote Indigenous Australians. Australian Journal of Rural Health, 15, 88-93. http://dx.doi.org/10.1111/j.1440-1584.2007.00869.x

[19] Cliford, A., Doran, C. and Tsey, K. (2013) A Systematic Review of Suicide Prevention Interventions Targeting Indigenous Peoples in Australia, United States, Canada and New Zealand. BMC Public Health, 13, 463. http://www.biomedcentral.com/1471-2458/13/463

[20] Wexler, L. and Gone, J. (2012) Culturally Responsive Suicide Prevention in Indigenous Communities: Unexamined Assumptions and New Possibilities. American Journal of Public Health, 102, 800-806. http://dx.doi.org/10.2105/AJPH.2011.300432

[21] Russel, L. (2013) Telling the Story of Maori Suicide Conference. Suicide Prevention 2013: A Life Span Perspective. The Ellerslie Events Centre, Auckland. 
Scientific Research Publishing (SCIRP) is one of the largest Open Access journal publishers. It is currently publishing more than 200 open access, online, peer-reviewed journals covering a wide range of academic disciplines. SCIRP serves the worldwide academic communities and contributes to the progress and application of science with its publication.

Other selected journals from SCIRP are listed as below. Submit your manuscript to us via either submit@scirp.org or Online Submission Portal.
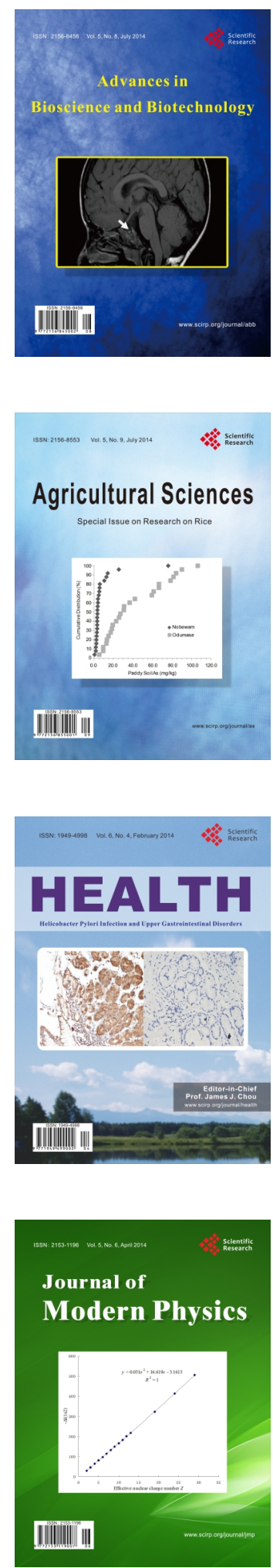
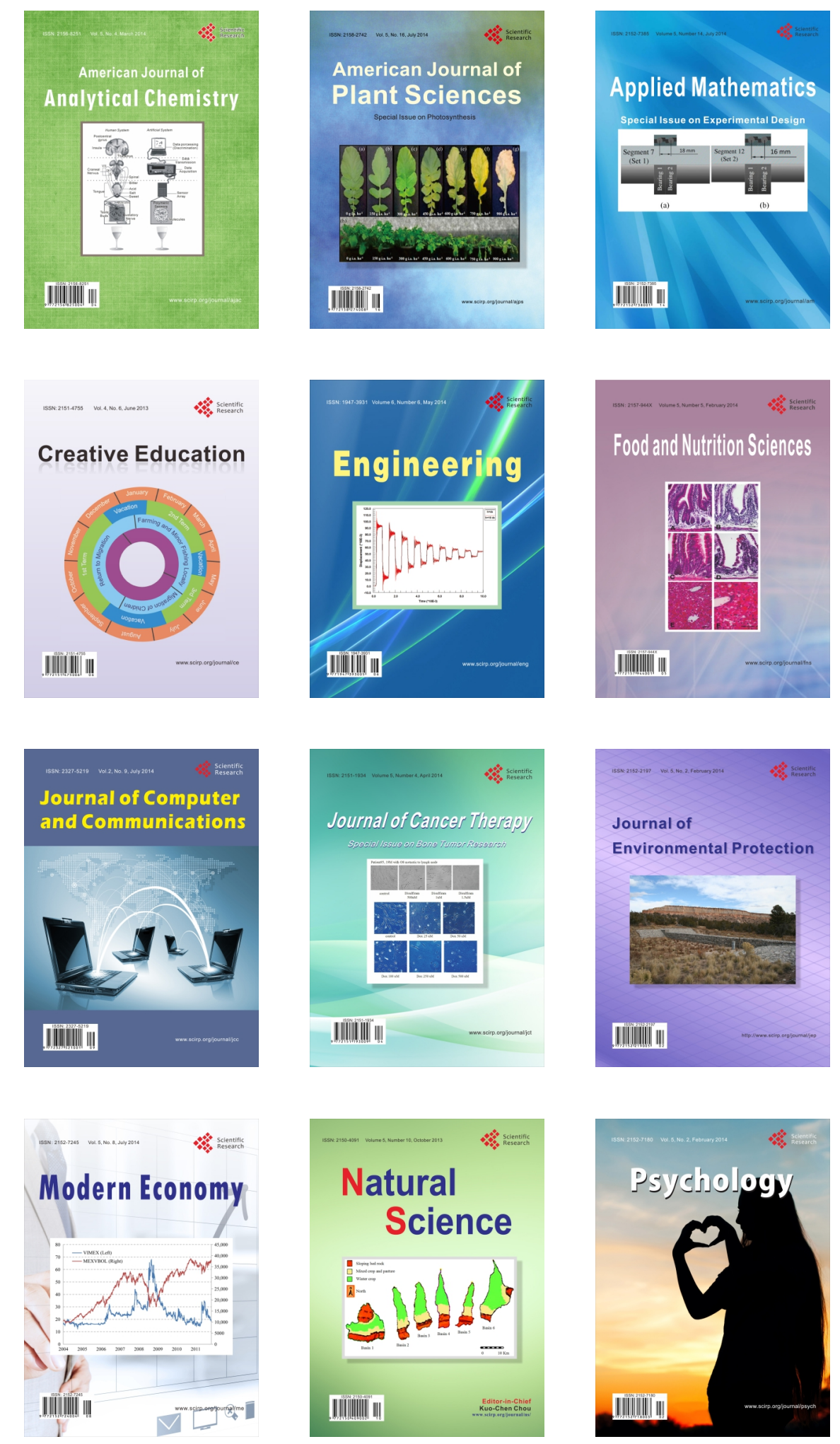